Vol. 16, Núm. 4

Oct.-Dic. 2020. pp 190-200

doi: $10.35366 / 97818$

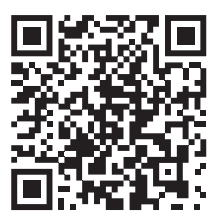

\title{
Tratamiento quirúrgico de las lesiones parciales del plexo braquial de nacimiento
}

\author{
Conservative treatment of birth-related brachial plexus injuries
}
Raul Emerico Rodríguez Martínez, * Marlene Vanessa Salcido Reyna, ${ }^{\ddagger}$ Mario Andre Chica Carpio,§ Jorge Francisco Clifton Correa, $"$ Leonardo López Almejo," Rene Humberto Barraza-Arrambide, ${ }^{* *}$ Ana Fernanda Espinosa de los Monteros-Kelley, ${ }^{\ddagger}$ Eduardo Pablo Zancolli, §§ Rogelio Josue Solano Pérez

\author{
* Cirujano Ortopedista, Cirujano de Mano, Cirugía de Nervio Periférico, Staff en \\ Hospital Puerta de Hierro Guadalajara. Diplomado de Cirugía de Plexo Braquial \\ UAdeC Torreón, Máster Brachial Plexus International School. \\ * Cirujano Ortopedista, Cirugía de Nervio Periférico, Staff en Hospital General de \\ Mexicali. Diplomado de Cirugía de Plexo Braquial UAdeC Torreón. \\ \& Cirujano Ortopedista, Cirujano de Mano, Cirugía de Nervio Periférico, Staff en Hospital \\ de Quito, Ecuador. Diplomado de Cirugía de Plexo Braquial UAdeC Torreón. \\ "Cirujano Plástico. Profesor de Cirugía de Plexo Braquial y Nervio Periférico. Hospital Universitario. \\ Universidad Autónoma de Coahuila. Profesor del Máster en Cirugía de Plexo Braquial BPIS. \\ " Cirujano Ortopedista, Cirujano de Mano, Cirugía de Nervio Periférico, Staffen \\ Star Médica Aguascalientes, Profesor del Diplomado de Cirugía de Plexo Braquial \\ UAdeC Torreón, Máster Brachial Plexus International School. \\ "Cirujano Ortopedista, Cirujano de Mano, Cirugía de Nervio Periférico, Staff \\ Christus Muguerza Hospital Saltillo, Profesor del Diplomado de Cirugía de Plexo \\ Braquial UAdeC Torreón, Máster Brachial Plexus International School. \\ \# Cirujano Ortopedista, Cirujano de Mano, Cirugía de Nervio Periférico, Staff en \\ Hospital Real San José Guadalajara Profesor del Diplomado de Cirugía de Plexo \\ Braquial UAdeC Torreón, Máster Brachial Plexus International School. \\ $\$ \$$ Cirujano Ortopedista, Cirujano de Mano, Cirugía de Nervio Periférico, Staff en \\ Hospital Fundación Favaloro Buenos Aires Argentina. Diplomado de Cirugía de Plexo \\ Braquial UAdeC Torreón, Máster Brachial Plexus International School. \\ "Cirujano Ortopedista, Cirujano de Columna, Cirugía de Nervio Periférico, Staff en \\ Hospital «Dr. Victorio de la Fuente Narváez», CDMX. Diplomado de Cirugía de Plexo \\ Braquial UAdeC Torreón, Máster Brachial Plexus International School. \\ Hospital Puerta de Hierro Andares, Guadalajara, Jalisco, México.
}

Correspondencia: Dr. Raúl Emérico Rodríguez Martínez. Blvd. Puerta de Hierro No. 5150, Torre A planta baja, 45118, Zapopan, Jalisco, México. Correo electrónico: dr.raulrodriguezm@gmail.com

Citar como: Rodríguez MRE, Salcido RMV, Chica CMA, Clifton CJF, López AL, Barraza-Arrambide RH y cols. Tratamiento quirúrgico de las lesiones parciales del plexo braquial de nacimiento. Ortho-tips. 2020; 16 (4): 190-200. https://dx.doi.org/10.35366/97818 


\section{RESUMEN}

Las lesiones del plexo braquial de nacimiento son lesiones relativamente comunes por el estiramiento del plexo braquial al momento del parto. Aproximadamente $30 \%$ de los pacientes no se recuperarán por completo y necesitarán de reparación quirúrgica. Se han realizado dos principales estrategias de tratamientos: la cirugía primaria, consistiendo en exploración y reconstrucción de la porción afectada del plexo braquial dentro de los primeros meses de vida del paciente, y los procedimientos secundarios que incluyen transferencias tendinosas 0 musculares, osteotomías y otros procedimientos ortopédicos. Los procedimientos secundarios pueden ser realizados como el único tratamiento de las lesiones del plexo braquial de nacimiento o después de la cirugía primaria para minimizar algún déficit funcional. Dos cosas son cruciales para obtener un buen resultado: la selección apropiada del paciente, separar aquellos que se recuperarán espontáneamente de aquellos que sólo se recuperarán parcialmente o no del todo; y una buena técnica quirúrgica.

Palabras clave: Lesión del plexo braquial, parálisis altas, plexo obstétrico, lesión perinatal, tratamiento quirúrgico.

\section{ABSTRACT}

Obstetric brachial plexus injuries are a relatively common stretch injury of the brachial plexus that occurs during delivery. Roughly $30 \%$ of patients will not recover completely and will need a surgical repair. Two main treatment strategies have been used: primary surgery, consisting in exploring and reconstructing the affected portions of the brachial plexus within the first few months of the patient's life, and secondary procedures that include tendon or muscle transfers, osteotomies, and other orthopedic techniques. Secondary procedures can be done as the only surgical treatment of Obstetric brachial plexus injuries or after primary surgery, in order to minimize any residual deficits. Two things are crucial to achieving a good outcome: the appropriate selection of patients, to separate those who will spontaneously recover from those who will recover only partially or not at all; and a good surgical technique.

Keywords: Brachial plexus injury, upper palsy, obstetric plexus, perinatal injury, surgical treatment.

\section{INTRODUCCIÓN}

La lesión del plexo braquial es definida como la debilidad flácida de la extremidad superior que ocurre como resultado de la tracción durante el nacimiento. ${ }^{1} \mathrm{La}$ incidencia reportada en varios países varía de entre 0.38 a 5.1 por 1,000 nacidos vivos; en Estados Unidos ha sido demostrada en 1.51 por cada 1,000 habitantes en datos obtenidos de 1997 a 2003. ${ }^{2}$ A medida que aumenta la incidencia de cesárea disminuye la incidencia de lesión del plexo braquial, el factor de riesgo más grande es la distocia de hombros, lo que significa que un niño tiene una probabilidad de $10.4 \%$ de sufrir una lesión del plexo si ocurre esta complicación durante el parto. La hipotonía es un factor de riesgo importante, aun así, en más de la mitad de los pacientes con lesión del plexo no existe un factor de riesgo previamente conocidos. En México no hay una incidencia conocida; sin embargo, se ha reportado de $0.37-0.87$ por cada 1,000 nacimientos; de acuerdo a los cubos dinámicos del sistema automatizado de egreso hospitalario se reportaron 45 casos a nivel nacional, de los cuales dos pertenecen a Jalisco en el año 2011. ${ }^{3}$ 
Históricamente, Smellie (1764) sugirió el origen obstétrico de una parálisis del brazo en niños. Duchenne de Boulogne (1872) describió a cuatro niños con lesión del plexo braquial superior como resultado de presentación de hombros en el parto. La descripción clásica de Erb (1874) se refería a la parálisis del plexo braquial superior en adultos que las mismas descritas por Duchenne de Boulogne. ${ }^{4}$ Thornburn (1903) propuso que la lesión del plexo resulta de una tracción excesiva durante el parto y en 1906 con su tesis demostró que puede ser el resultado de un estiramiento excesivo, cuando el hombro se impacta en la sínfisis del pubis; de diferente forma, en la presentación de nalgas, la lesión ocurre por dificultades, porque el brazo queda extendido y atrapado quedando una fuerza de tracción combinada con un movimiento lateral. ${ }^{4}$

Existen muchos estudios sobre la lesión parcial del plexo braquial, se estima que la recuperación neurológica espontánea se reporta en 66 a 92\% de los infantes. El pronóstico de recuperación depende de la extensión de las raíces involucradas y la severidad del nervio lesionado, un estimado de $80 \%$ de los infantes con lesión C5-C6 recuperarán espontáneamente la función, en comparación de 60\% de recuperación que tendrán aquellos con lesión C5-C7. El pronóstico de recuperación para infantes con lesión de C5-T1 sin síndrome de Horner asociado es menor a $50 \%$ y para quienes tienen síndrome de Horner asociado es de $10 \%$.

La presencia del síndrome de Horner es un importante signo de mal pronóstico y está relacionado a una lesión extensa con avulsión de las raíces bajas. La probabilidad de recuperación es tan pobre que Narakas lo clasificó en el grupo 4 de lesiones del plexo braquial obstétrico y en estos infantes está indicada la cirugía tempranamente para la reconstrucción microquirúrgica del plexo braquial menor a tres meses.

Un factor pronóstico en la recuperación del plexo braquial es la flexión del codo en contra de la gravedad y puede ser usado como guía de tratamiento para la toma de decisiones. Los infantes que no recuperen la flexión del codo en contra de la gravedad a los tres meses tienen menor probabilidad de recuperación y el incremento del tiempo para la recuperación de flexión del codo está asociada a una pobre recuperación funcional.

De acuerdo a la evaluación motora podemos dividir las lesiones en: a) parálisis de raíces altas: la extremidad superior se encuentra en rotación interna y pronación, la abducción activa no es posible, el codo está ligeramente flexional (en parálisis C5-C7) o completa-
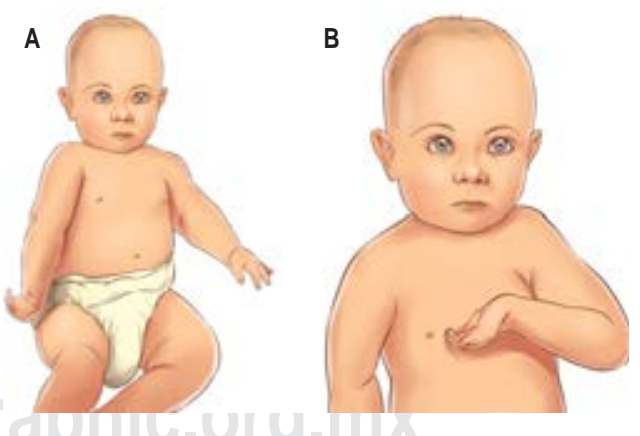

Figura 1: A) Parálisis Erb-Duchenne (lesión de raíces altas).

B) Parálisis Dejerine-Klumke (lesión de raíces bajas). Tomado de: Socolovsky M, Nizzo G. Obstetric brachial plexus palsy: reviewing the literature comparing the results of primary versus secondary surgery. Childs Nerv Syst. 2016; 32 (3): 415-425. 
mente extendido (parálisis C5-C6) y la muñeca y los dedos están flexionados; y b) parálisis completa, la extremidad superior está flácida y la mano se encuentra sin ningún tono ${ }^{5}$ (Figura 1).

Las lesiones del plexo braquial se clasifican de acuerdo con su localización anatómica (como supraclavicular, retroclavicular o infraclavicular), el nivel de lesión (plexo alto o plexo bajo) y la ubicación del nervio espinal (como avulsión o ruptura) ${ }^{6}$ (Figura 2).

\section{INDICACIONES QUIRÚRGICAS}

La intervención microquirúrgica busca mejorar la función, a menudo, sin la expectativa de que la extremidad afectada se recuperará completamente. El consenso general es que la microcirugía reconstructiva debería realizarse en menores con lesiones totales y con síndrome de Horner a la edad de tres meses. ${ }^{7}$ Sin una intervención quirúrgica estos pacientes tienen un déficit funcional importante de por vida. Una cirugía a edad temprana es importante en lesiones totales para minimizar la pérdida de placas neuro-
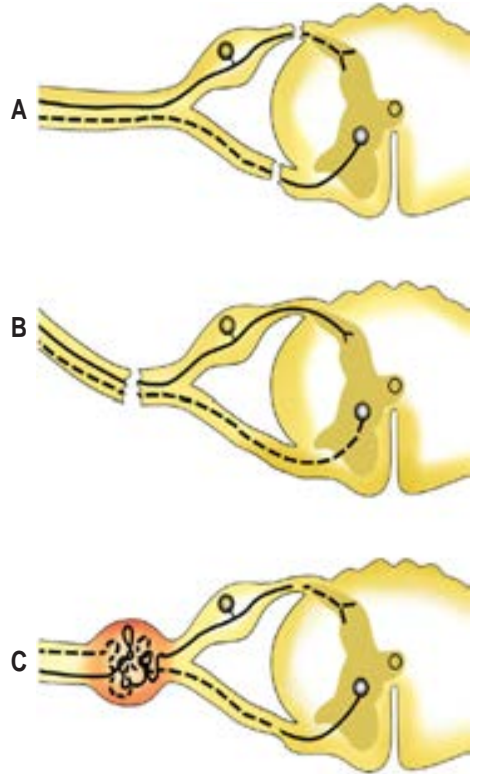

Figura 2: A) Avulsión de raíz.

B) Ruptura. C) Lesión por elongación.

Tomado de: Socolovsky M, Nizzo G.

Obstetric brachial plexus palsy: reviewing the literature comparing the results of primary versus secondary surgery. Childs Nerv Syst. 2016; 32 (3): 415-425.

musculares y para maximizar el tiempo de recuperación. Existen argumentos de que el criterio para una intervención temprana debería expandirse para incluir pacientes con lesiones totales desde su inicio con recuperación de bíceps, pero sin recuperación significativa de la función de la mano y el antebrazo. ${ }^{8}$ Por el contrario, pacientes con lesiones altas del plexo que logran recuperar bíceps a los tres meses deben ser tratados sin cirugía.

El factor más controversial en el tratamiento de las LPBN consiste en qué momento operar las lesiones de tipo elongación o ruptura, en las cuales existe variedad en la severidad de la lesión y grado de recuperación. Gilbert y Tassin, ${ }^{9}$ así como otros cirujanos, ${ }^{10,11}$ adoptaron la ausencia de función del bíceps a los tres meses como indicación para la intervención microquirúrgica. Ellos refieren haber tenido más resultados pobres en la función del hombro a los cinco años e incremento de la probabilidad de mejoría con procedimientos secundarios en pacientes que recuperaron la función del bíceps después de tres meses. Otros autores han adoptado un manejo más conservador al referir que la ausencia de la flexión sólo de codo a los tres meses puede ser un predictor incorrecto de una pobre recuperación y llevar a procedimientos quirúrgicos innecesarios. ${ }^{12,13}$ Estos autores reportan que los pacientes que recuperan la función del bíceps entre los cuatro y seis meses de edad fueron capaces de alcanzar una función 
global del hombro con transferencias tendinosas secundarias, ${ }^{12,13}$ comparada con la función en aquellos que se les realizó cirugía a los tres meses de edad. En otro estudio, la recuperación del bíceps entre los cinco y seis meses pareció el mejor resultado, ${ }^{12}$ pero, aun así, el tiempo exacto para realizar la cirugía sigue siendo desconocido.

La cirugía debe ser indicada entre los tres y seis meses de edad en las lesiones extraforaminales. Clark y Curtis, de manera rutinaria, usan la recuperación del bíceps a los nueve meses como determinante para realizar o no la intervención quirúrgica. ${ }^{14}$ La capacidad del niño de llevar una galleta a la boca (cookie test) es un factor decisivo para guiar el tratamiento. Chuang y cols. realizaron una revisión retrospectiva de 78 menores con cuatro años de seguimiento y determinaron que la mejoría de la función del hombro y codo era similar para pacientes tratados quirúrgicamente inicialmente o después. Sin embargo, la función de la mano fue pobre cuando la cirugía fue realizada posterior a la infancia. ${ }^{15}$ Grossman y cols. reportaron en una serie pequeña de menores que fueron sometidos a cirugía después de recuperar la función del bíceps a los 10 meses de edad o después. El objetivo de la intervención quirúrgica en este pequeño grupo fue aumentar la abducción y rotación externa del hombro, incluso después de la recuperación neuronal parcial. Las técnicas quirúrgicas realizadas en esta serie incluyen injertos nerviosos y transferencias nerviosas al nervio supraescapular, con buenos resultados reportados en todos los niños. ${ }^{16}$

\section{INDICACIONES QUIRÚRGICAS Y TIMING EN NUESTRA PRÁCTICA (Tabla 1):}

\section{Tabla 1: Reconstrucción microquirúrgica y/o transferencias nerviosas.}

Tres a seis meses sin recuperación de bíceps "cookie test»

Lesiones severas:

- Parálisis total

- Horner (+)

- No recuperación de mano

Cirugía entre las ocho a 10 semanas

No realizamos estudios de imagen de manera rutinaria

EMG no es solicitada de rutina

Exploración física para valorar recuperación es la herramienta más confiable

\section{MANEJO QUIRÚRGICO}

\section{Manejo quirúrgico nervioso}

El tratamiento de las LPBN se puede dividir en cirugía de nervio primaria o tardía. Las primarias son aquellas que se realizan dentro de los primeros dos años de vida y tienen como objetivo primordial llevar el mayor aporte axonal a esos músculos paralizados, esto para lograr la mayor función posible y minimizar las 
secuelas propias de la lesión. La cirugía de nervio tardía es aquella que se realiza después de dos años de vida y tiene como objetivo principal tratar secuelas para mejorar la función de la extremidad y reinervar músculos específicos a través de transferencias nerviosas.

- Cirugía de nervio primaria (reconstrucción nerviosa \pm transferencias nerviosas o coaptación directa).

- Cirugía de nervio tardía (transferencias nerviosas y musculares, liberación de contracturas musculares y articulares, coracoidectomía, osteotomías, etcétera).

\section{Cirugía de nervio primaria}

Muchos de los avances recientes en el tratamiento microquirúrgico de las LPBN han sido en técnicas para la reinervación de la musculatura de la extremidad superior. El espectro de cirugía de nervio históricamente incluye neurólisis, resección de neuroma y reconstrucción con injerto nervioso. Las transferencias nerviosas ${ }^{17}$ y los conductores nerviosos han llevado a una expansión de los procedimientos disponibles para la reconstrucción nerviosa. La neurólisis como tratamiento único no está más indicada en las LPBN. ${ }^{18}$

El hallazgo quirúrgico más común en LPBN es un neuroma en continuidad del tronco superior en la unión de las raíces C5 y C6. Clark y cols. reportan mejores resultados a largo plazo con resección del neuroma e injertos nerviosos comparado a neurólisis únicamente. ${ }^{18}$ Después de la resección del neuroma, la reparación directa es raramente realizada debido a la dificultad de la coaptación directa de nervios sanos sin tener tensión; sin embargo, algunos cirujanos continúan usando esta técnica en la que describen favorables resultados en pacientes jóvenes. ${ }^{19}$ Los injertos nerviosos puentean el tejido nervioso viable de proximal a distal a la lesión. El nervio sural autólogo es comúnmente utilizado, aunque pueden ser utilizados otros injertos donadores del mismo miembro afectado. La resección del neuroma y la reconstrucción con injertos nerviosos son el estándar de oro del tratamiento microquirúrgico para las lesiones por ruptura, ${ }^{20}$ de las cuales otras técnicas necesitan ser comparadas.

Las transferencias nerviosas son cada vez más comunes en el tratamiento quirúrgico de las LPBN. Éstas ahora están siendo utilizadas durante cirugías primarias en lugar de, o en conjunto, con reconstrucción con injertos nerviosos y en casos tardíos para aumentar la función en lesiones parcialmente recuperadas. A diferencia de los injertos nerviosos, las transferencias nerviosas tienen el beneficio de que un solo procedimiento quirúrgico brinda un donador motor a motor directo.

Las transferencias nerviosas fueron inicialmente descritas para mejorar la función del bíceps y del manguito rotador. La función del infraespinoso y supraespinoso puede ser restaurada de manera directa al transferir la rama motora terminal del nervio espinal accesorio al nervio supraescapular. En el caso de las lesiones del tronco superior con escasez de axones viables, la 
transferencia para el nervio supraescapular se realiza de forma común. ${ }^{21} \mathrm{La}$ reinervación del deltoides y los músculos del manguito rotador pueden llevar a un mejor resultado de la función del hombro que sólo la reinervación del supraespinoso. Al emplear la transferencia del espinal accesorio en conjunto con el uso de la rama motora de la porción larga del tríceps del nervio radial y al transferirla a la porción anterior del nervio axilar, se obtiene una mejoría en la abducción por encima de sólo reinervar el nervio supraescapular. ${ }^{22}$ Oberlin y cols. fueron los primeros en describir la transferencia de los fascículos del nervio cubital que inervan al flexor carpi ulnaris como donadoras para las ramas motoras del bíceps. Al-Qattan utilizó esta técnica en LPBN con éxito, así como otros. Adaptando esta técnica, los fascículos motores del nervio mediano han sido también utilizados para reinervar el bíceps o el músculo braquial. Los nervios intercostales torácicos han sido también descritos para restaurar la flexión del codo, como también se tiene el nervio espinal accesorio. La transferencia del nervio pectoral medial para el nervio musculocutáneo ha sido también descrita en niños con parálisis de nacimiento, con 70 a $80 \%$ de pacientes con recuperación funcional. Con la presencia de una raíz nerviosa $\mathrm{C} 7$ ipsilateral intacta, la transferencia de esa raíz C7 para reinervar aisladamente el tronco superior lesionado ha sido utilizado con éxito. De manera adicional, la rama $\mathrm{C} 7$ contralateral ha sido utilizada en lesiones por avulsiones extensas en un intento por recuperar tanto la flexión del codo como la función de la mano, aunque se ha reportado persistencia de la sincronicidad de movimiento del lado no afectado. Estas transferencias de fascículos motores de nervios sanos extraplexales para reinervar ramas motoras de músculos paralizados han incrementado de manera importante las opciones de tratamiento microquirúrgico. Las transferencias no sólo proveen una excelente opción para cirugía primaria, sino también ofrecen opciones para microcirugía tardía, transferencias tendinosas regionales o reconstrucción con músculos funcionales libres para niños que no tuvieron una recuperación, tratados con o sin cirugía previa. ${ }^{23}$

La coaptación directa es una opción más dentro de las técnicas de reconstrucción microquirúrgica, consiste en resección de neuroma, acercar ambos cabos, distal como proximal y realizar sutura directa. Esta técnica tiene claras ventajas, no se necesitan injertos, es rápida (y probablemente más fuerte), recuperación mediante el aporte de una mayor densidad fascicular y una reconstrucción «anatómica». Los inconvenientes son que el cirujano debe aceptar cierta tensión en el sitio de la coaptación (codo flexional a $90^{\circ}$ ) y algunos neuromas fasciculares a nivel de la sección de los nervios y de la sutura, lo cual puede ser causa de coactivaciones neuromatosas. ${ }^{24}$ Las limitaciones de esta técnica son la distancia entre ambos nervios y la disección distal para ganar más distancia, logrando una sutura con menos tensión.

Las posibilidades de realizar transferencias de nervios en combinación con injertos nerviosos o suturas directas, permite que las probabilidades de restauración de la función aumenten cada vez. En cada caso de LPBN, el plan debe ser individualizado, basado en la locación y extensión de la lesión. ${ }^{25}$ 


\section{Cirugías secundarias}

Las deformidades residuales son clásicas, incluyen discrepancias de crecimiento de los huesos en el lado afectado. Las deformidades más frecuentes incluyen contractura en rotación interna del hombro (72\%), contractura en flexión de codo $(62 \%)$, contractura en supinación del antebrazo (69\%), desviación cubital de la muñeca $(27 \%)$.

Los procedimientos secundarios se realizan cuando los pacientes dejan de tener mejoría y permanecen estancados a pesar de los esfuerzos en rehabilitación, por lo general a los 24 meses de edad. Los procedimientos incluyen transferencias de nervio distales, transferencias musculares, liberaciones musculares o tenotomías, transferencias de músculos funcionales libres o pediculados y procedimientos óseos.

\section{LESIÓN C5-C6, EXTRAFORAMINAL (RUPTURA) CON DOS RAÍCES DISPONIBLES}

Lo primero es comprobar la integridad del nervio torácico largo que estimula al serrato anterior y al nervio dorsal escapular que inerva el músculo romboides, eso corrobora la indemnidad de las raíces disponibles para la reconstrucción, ya que son nervios que emergen directamente de las raíces antes de convertirse en tronco primario superior (TPS). Para la reconstrucción se utiliza injerto de nervio autólogo y se conectan cables desde C5 y C6 hacia la porción del nervio supraescapular, división anterior y división posterior del TPS (Figura 3).
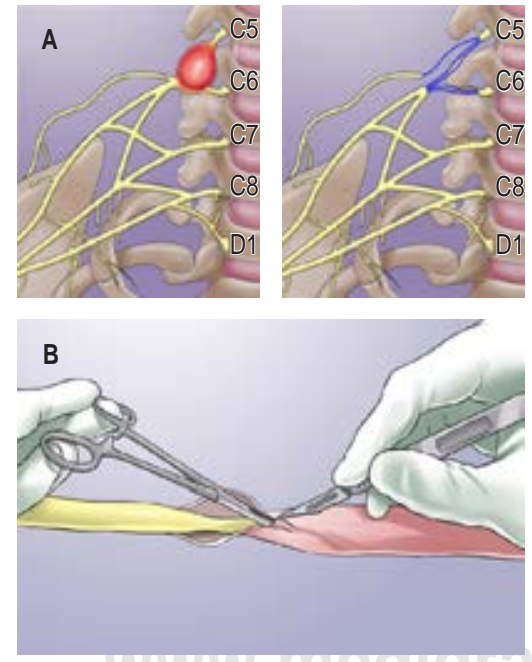

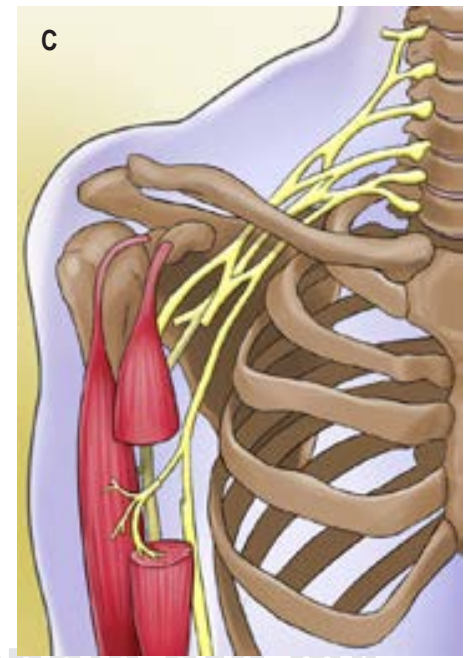

Figura 3: A) Neuroma en continuidad en tronco primario superior (TPS) a la izquierda, y a la derecha reconstrucción de C5 y C6 con injerto de nervio sural para división anterior, división posterior y nervio supraescapular. B) Neurólisis es la liberación de tejido fibrótico alrededor del nervio. C) Ejemplo de transferencia nerviosa de cubital (fascículo de FCR) para musculocutáneo (rama de bíceps) técnica de Oberlin.

Tomado de: Socolovsky M, Nizzo G. Obstetric brachial plexus palsy: reviewing the literature comparing the results of primary versus secondary surgery. Childs Nerv Syst. 2016; 32 (3): 415-425. 


\section{LESIÓN C5-C6 CON UNA RAÍZ DISPONIBLE}

Si bien es cierto, las fibras axonales de $\mathrm{C} 5$ tienen menor número que las fibras de C6, ante una lesión alta con una avulsión de una de ellas, el peor pronóstico es la avulsión de C6. La reconstrucción se realizará mediante neurotización del nervio espinal accesorio al nervio supraescapular y mediante injerto autólogo se llevará a cabo la reconstrucción con cables desde la porción proximal de la raíz disponible hacia el tronco primario superior a sus divisiones anterior y posterior.

\section{LESIÓN C5-C6 SIN NINGUNA RAÍZ DISPONIBLE}

El peor de los escenarios es tener una lesión alta de C5-C6 sin raíces disponibles para la reconstrucción. En prioridad se define tener función de codo antes que de hombro, en ese orden, la opción que tenemos es la transferencia nerviosa utilizando donadores extraplexales, nervio espinal accesorio (XI) a nervio supraescapular, Oberlin para dar flexión de codo y nervio radial (rama para tríceps porcino larga) para inervar deltoides. Otra opción de nervios donadores son los intercostales.

\section{LESIÓN C5, C6, C7}

El problema de estos pacientes, al igual que las lesiones de raíces altas, son una buena mano con una buena flexión de dedos y de pulgar y/o una débil extensión de dedos. Las prioridades son función de codo en primer lugar, segundo la función del hombro, y, por último y no menos importante, la extensión de muñeca.

\section{LESIONES C5, C6, C7 CON TRES RAÍCES DISPONIBLES}

El mejor escenario es disponer de todas las raíces integras, es necesario la reconstrucción anatómica con múltiples injertos de nervio, múltiples cables de aproximadamente $3 \mathrm{~cm}$ cada uno. Se conectarán las raíces C5-C6 al tronco primario superior y un injerto para nervio espinal accesorio, y la raíz $\mathrm{C} 7$ al tronco primario medio. Este procedimiento tiene buenos resultados en la función del paciente cuando son realizados de manera temprana.

\section{LESIONES C5, C6, C7 CON DOS O UNA RAÍZ DISPONIBLE}

Si disponemos de dos raíces la reconstrucción se hace con la raíz de C5 para división posterior y C6 por su mayor aporte de fibras mielínicas para división anterior mediante el uso de injerto de nervio autólogo, más la neurotización del nervio espinal accesorio al nervio supraescapular. Si se dispone de una raíz disponible es preferible contar con la raíz de C6, en el mismo escenario se realiza la reconstrucción de la única raíz para la división posterior y división anterior, el uso de injerto autólogo es imperativo y la transferencia del nervio espinal accesorio al nervio supraescapular de igual forma; aunque la distribución de fibras motoras 
hacia las fibras distales será pobre, el resultado de la reinervación puede quedar limitado a una menor función.

\section{LESIONES BAJAS}

Las lesiones bajas son producidas por una abducción forzada del hombro, principalmente en partos distócicos de nalgas. Las raíces más vulnerables son C8 y $\mathrm{T} 1$, teniendo como resultado una parálisis en mano. En estos casos la prioridad es restaurar la función de la mano, reinervando el tronco primario inferior (TPI); si se cuenta con alguna raíz disponible se debe hacer una reconstrucción con injerto de nervio autólogo para TPI, en caso de no contar con raíces disponibles, se puede usar como donador la raíz de $\mathrm{C} 7$, sacrificando la raíz para hacer reconstrucción con injerto de nervio para el TPI.

\section{CONCLUSIÓN}

Los menores que han sufrido una LPBN tienen una amplia variedad de presentaciones clínicas, sus cuidados y tratamientos deben adaptarse de acuerdo a su lesión. Desarrollos recientes en el manejo de nervio, tejidos blandos y procedimientos óseos han permitido mejores resultados funcionales. A pesar de estos avances, el momento apropiado de la intervención quirúrgica y el tipo de procedimiento en menores que no han recuperado ninguna función espontáneamente, continúa siendo un tema de controversia, en nuestra práctica dejamos en claro el timming quirúrgico para las diferentes presentaciones de lesiones.

\section{BIBLIOGRAFÍA}

1. Defrancesco CJ, Shah DK, Rogers BH, Shah AS. The epidemiology of brachial plexus birth palsy in the united states: declining incidence and evolving risk factors. J Pediatr Orthop. 2019; 39 (2): e134-e140.

2. Abzug JM, Mehlman CT, Ying J. Assessment of current epidemiology and risk factors surrounding brachial plexus birth palsy. J Hand Surg. 2019; 44 (6): 515.e1-515.e10.

3. Diagnóstico y tratamiento de la lesión Obstétrica del plexo braquial en niños y niñas de 0 a 15 años en el 2do Nivel de Atención. México, Secretaría de Salud 2002.

4. Gilbert A. Brachial plexus injuries. Martin Dunitz Ltd, 2001, p. 151.

5. Examination C. Long-term results of primary repair of brachial plexus lesions in children. Microsurgery. 2006; 26 (4): 334-342.

6. Borrero Brachial plexus overwiew. making it simple.

7. Water PM. Obstetric brachial plexus injuries: evaluation and management. J Am Acad Orthop Surg. 1997; 5: 205-214.

8. Haerle M, Gilbert A. Management of complete obstetric brachial plexus lesions. J Pediatr Orthop. 2004; 24: 194-200.

9. Gilbert A, Tassin JL. Surgical repair of the brachial plexus in obstetric paralysis. Chirurgie. 1984; 110: 70-75.

10. Bain JR, Dematteo C, Gjertsen D, Hollenberg RD. Navigating the gray zone: a guideline for surgical decision making in obstetrical brachial plexus injuries. J Neurosurg Pediatr. 2009; 3: 173-180.

11. Terzis JK, Kokkalis ZT. Shoulder function following primary axillary nerve reconstruction in obstetrical brachial plexus patients. Plast Reconstr Surg. 2008; 122: 1457-1469.

12. Waters PM. Comparison of the natural history, the outcome of microsurgical repair, and the outcome of operative reconstruction in brachial plexus birth palsy. J Bone Joint Surg. 1999; 81A: 649-659.

13. Al-Qattan MM. The outcome of Erb's palsy when the decision to operate is made at 4 months of age. Plast Reconstr Surg. 2000; 106: 1461-1465. 
14. Clarke HM, Curtis CG. An approach to obstetrical brachial plexus injuries. Hand Clin 1995; 11: 563-580; discussion 580-581.

15. Chuang DC, Mardini S, Ma HS. Surgical strategy for infant obstetrical brachial plexus palsy: experiences at Chang Gung memorial hospital. Plast Reconstr Surg. 2005; 116: 132-142; discussion 143-144.

16. Grossman JA, DiTaranto P, Yaylali I, Alfonso I, Ramos LE, Price AE. Shoulder function following late neurolysis and bypass grafting for upper brachial plexus birth injuries. J Hand Surg. 2004; 29B: 356-358.

17. El-Gammal TA, Fathi NA. Outcomes of surgical treatment of brachial plexus injuries using nerve grafting and nerve transfers. J Reconstr Microsurg. 2002; 18: 7-15.

18. Clarke HM, DiTaranto P, Yaylali I, Alfonso I, Ramos LE, Price AE. Obstetrical brachial plexus palsy: results following neurolysis of conducting neuromas-in-continuity. Plast Reconstr Surg. 1996; 97: 974982; discussion 983-984.

19. Kirjavainen M, Remes V, Peltonen J, Kinnunen P, Pöyhiä T, Telaranta T, et al. Long-term results of surgery for brachial plexus birth palsy. J Bone Joint Surg. 2007; 89A: 18-26.

20. Waters PM, Bae DS. Effect of tendon transfers and extra-articular soft-tissue balancing on glenohumeral development in brachial plexus birth palsy. J Bone Joint Surg. 2005; 87A: 320-325.

21. Terzis JK, Kokkalis ZT. Shoulder function following primary axillary nerve reconstruction in obstetrical brachial plexus patients. Plast Reconstr Surg. 2008; 122: 1457-1469.

22. Bertelli JA, Ghizoni MF. Reconstruction of $\mathrm{C} 5$ and $\mathrm{C} 6$ brachial plexus avulsion injury by multiple nerve transfers: spinal accessory to suprascapular, ulnar fascicles to biceps branch, and triceps long or lateral head branch to axillary nerve. J Hand Surg. 2004; 29A: 131-139.

23. Waters PM. Current concepts in the management of brachial plexus birth palsy. J Hand Surg. 2010; 35A: 322-330.

24. Foad SL, Mehlman CT, Ying J. The epidemiology of neonatal brachial plexus palsy in the United States. J Bone Joint Surg. 2008; 90A: 1258-1264.

25. Gilbert A. 1996 the newborn palsies. In: Alnot J. Narakas A. eds: traumatic Brachial Plexus Injuries. 